\title{
Fast Fourier Transform Based Power Option Pricing with Stochastic Interest Rate, Volatility, and Jump Intensity
}

\author{
Jiexiang Huang, Wenli Zhu, and Xinfeng Ruan \\ School of Economic Mathematics, Southwestern University of Finance and Economics, Chengdu 611130, China \\ Correspondence should be addressed to Jiexiang Huang; huangjiex@gmail.com
}

Received 21 August 2013; Revised 27 October 2013; Accepted 28 October 2013

Academic Editor: Ram N. Mohapatra

Copyright ( 2013 Jiexiang Huang et al. This is an open access article distributed under the Creative Commons Attribution License, which permits unrestricted use, distribution, and reproduction in any medium, provided the original work is properly cited.

\begin{abstract}
Firstly, we present a more general and realistic double-exponential jump model with stochastic volatility, interest rate, and jump intensity. Using Feynman-Kac formula, we obtain a partial integrodifferential equation (PIDE), with respect to the moment generating function of log underlying asset price, which exists an affine solution. Then, we employ the fast Fourier Transform (FFT) method to obtain the approximate numerical solution of a power option which is conveniently designed with different risks or prices. Finally, we find the FFT method to compute that our option price has better stability, higher accuracy, and faster speed, compared to Monte Carlo approach.
\end{abstract}

\section{Introduction}

In financial markets, option pricing problem becomes important for market practitioners ranging from individuals to financial institutes. The classic methodology for option pricing is developed by Black and Scholes [1]. In their model, the stock price follows a geometric Brownian motion and the option pricing formula in no arbitrage. However, a large number of empirical examples show that the initial assumption of their model generates high pricing deviations between theory and practice, for example the volatility clustering in [2] and the incompleteness of markets in [3]. Due to those drawbacks, some market practitioners begin to propose new underlying asset processes to fit empirical facts and develop new option types to control and reduce risks.

Double-exponential jump model with stochastic volatility, interest rate, and jump intensity could overcome these drawbacks of the Black-Scholes model in [2-8]. For example, Santa-Clara and Yan $[4,5]$ find that innovations to two risks, respectively, denoted by diffusion volatility process and jump intensity process in their model, have affected the expected return in the stock market. The empirical results show that those two processes are largely uncorrelated and do not support models that make jump intensity vary with the level of diffusive volatility. Based on the results of their theory, we believe that two components of risk are more conducive to the optimization of our model. An excellent contribution of our model is developing the model of Santa-Clara and Yan in $[4,5]$, combing stochastic volatility, interest rate, and meanreverting jump intensity processes. Thus, our model better corresponds with the real market than other constant jump intensity models.

As for new option types, compared with other exotic options, power option with considerable operability is simpler and more convenient in practical financial market [9]. Unfortunately, research on power option pricing is literally nil according to our best knowledge. Therefore, in this paper, we define a power option and creatively study and explain how the power coefficient affects the valuation of option.

However, it is difficult to get an exact solution expression of option price. Several numerical methods have been proposed to solve it, including finite difference methods by Zhang and Wang in [10,11], Monte Carlo simulation by Green in [12] and Glasserman in [13] and the FFT method by Carr and Madan in [14]. However, the finite difference method and Monte Carlo simulation are difficult to be applied in option pricing because they require substantially more computing time [14-17]. Compared to those approaches, the FFT is more effective and simpler to compute in [14-17]. Therefore, in this paper, we employ the FFT to compute the valuation of power potion. 
In conclusion, the prominent contribution in our paper is modeling a more general and realistic model with stochastic interest rate, volatility, and jump intensity. Defining the power option, discussing the effects of power coefficient, and using the FFT to obtain its numerical results are another three contributions.

This paper proceeds as follows. In Section 2, we build our model which links double-exponential jump, stochastic interest rate, volatility, and jump intensity. The characteristic function is derived in Section 3. In Section 4, we discuss the approximate solutions for power option pricing by the FFT. Numerical examples and discussions of the power coefficient are shown in Section 5. Conclusions are in Section 6.

\section{Our Model}

Let $\left(\Omega, \mathscr{F}_{t}, \mathbb{Q}\right)$ be a probability space where $\mathscr{F}_{t}$ is the filtration generated by the Brownian motion and jump process at time $t, 0 \leq t \leq T$ and $\mathbb{Q}$ is a risk-neutral probability. The underlying asset price $S(t)$ at time $t$ is given by

$$
\begin{aligned}
d S(t) / S(t)= & (r(t)-\lambda(t) m) d t+\sqrt{V(t)} d W^{s}(t) \\
& +\left(e^{y}-1\right) d N^{s}(t), \quad S(0)=S_{0}>0, \\
d r(t)= & \kappa_{r}\left(\theta_{r}-r(t)\right) d t \\
& +\varepsilon_{r} \sqrt{r(t)} d W^{r}(t), \quad r(0)=r_{0}>0, \\
d V(t)= & \kappa_{v}\left(\theta_{v}-V(t)\right) d t \\
& +\varepsilon_{v} \sqrt{V(t)} d W^{v}(t), \quad V(0)=V_{0}>0, \\
d \lambda(t)= & \kappa_{\lambda}\left(\theta_{\lambda}-\lambda(t)\right) d t \\
& +\varepsilon_{\lambda} \sqrt{\lambda(t)} d W^{\lambda}(t), \quad \lambda(0)=\lambda_{0}>0,
\end{aligned}
$$

where $W^{s}(t), W^{r}(t), W^{v}(t)$, and $W^{\lambda}(t)$ are Brownian motions; the correlation coefficient of $W^{s}(t)$ and $W^{V}(t)$ is $\rho>0$, and other motions are independent on each other; $N^{s}(t)$ is a Poisson process with stochastic intensity $\lambda(t)$, and jump size $y$ is a random variable; $m=E^{\mathbb{Q}}\left[e^{y}-1\right]$ is average jump amplitude; $r(t)$ is the stochastic interest rate and $V(t)$ is stochastic volatility of the underlying asset return; the parameters $\varepsilon_{r}, \varepsilon_{v}$, and $\varepsilon_{\lambda}$ and the mean-reverting rates $\kappa_{r}$, $\kappa_{v}$, and $\kappa_{\lambda}$ are positive constants; the constants $\theta_{r}, \theta_{v}$, and $\theta_{\lambda}$ are the long-term interest rate, volatility, and jump intensity, respectively.

We suppose that jump size $y$ has an asymmetric double exponential distribution $f(y)$,

$$
\begin{aligned}
f(y)= & p \frac{1}{\eta_{u}} e^{\left(-1 / \eta_{u}\right) y} 1_{y \geq 0} \\
& +q \frac{1}{\eta_{u}} e^{\left(1 / \eta_{d}\right) y} 1_{y<0}, \quad 0<\eta_{u}<1, \eta_{d}>0,
\end{aligned}
$$

where $p, q \geq 0, p+q=1$ are probability of up-move jump and down-move jump, respectively. $1 / \eta_{u}$ and $1 / \eta_{d}$ are mean of positive jumps and negative jumps, respectively. Therefore, $m=p /\left(1-\eta_{u}\right)+q /\left(\eta_{d}+1\right)-1$.

\section{The Characteristic Function}

Given the log asset price process in (1), it is possible to obtain the characteristic function $\phi(\cdot)$ of $X(\tau)$ at time $\tau=$ $T-t$. Under the risk-neutral measure $\mathbb{Q}$, firstly, we define an explicit expression of the moment generating function (MGF) $M(\Phi, X, r, V, \lambda, \tau)$ of $X(\tau)$ at time $\tau$,

$$
M(\Phi, x, r, V, \lambda, \tau)=\mathrm{E}^{\mathbb{Q}}\left[e^{\Phi X(\tau)}\right]=e^{-r \tau} \mathrm{E}^{\mathbb{Q}}\left[e^{r \tau} e^{\Phi X(\tau)}\right],
$$

and the complex-valued characteristic function is given by $\phi(u)=M(i u)$.

From (3), we find that $M(\Phi, X, r, V, \lambda, \tau)$ can be interpreted as a contingent claim that pays off $e^{r \tau+\Phi X(t)}$ at time $\tau$. Therefore, it is possible to get the MGF $M(\Phi, X, r, V, \lambda, \tau)$ and then obtain the characteristic function $\phi(u)$ in following theorem.

Theorem 1. The characteristic function $\phi(u)$ of $X(\tau)$ under the measure $\mathbb{Q}$ is given by

$$
\phi(u)=e^{i u X+i u(r-d) \tau+A(u, \tau)+B(u, \tau) V+C(u, \tau)+D(u, \tau) \lambda},
$$

where

$$
\begin{gathered}
A(u, \tau)=-\frac{\kappa_{v} \theta_{v}}{\varepsilon_{v}^{2}}\left(\varphi_{+} \tau+2 \ln \left[\frac{\varphi_{-}+\varphi_{+} e^{-\varsigma \tau}}{2 \varsigma}\right]\right), \\
B(u, \tau)=-\left(i u+u^{2}\right) \frac{1-e^{-\varsigma \tau}}{\varphi_{-}+\varphi_{+} e^{-\zeta \tau}}, \\
C(u, \tau)=-\frac{\kappa_{\lambda} \theta_{\lambda}}{\varepsilon_{\lambda}^{2}}\left(\psi_{+} \tau+2 \ln \left[\frac{\psi_{-}+\psi_{+} e^{-\xi \tau}}{2 \xi}\right]\right), \\
D(u, \tau)=2 \Lambda(u) \frac{1-e^{-\xi \tau}}{\psi_{-}+\psi_{+} e^{-\xi \tau}}, \\
\varphi_{ \pm}=\mp\left(\kappa_{v}-i u \rho \varepsilon_{v}\right)+\varsigma \\
\sqrt{\left(\kappa_{v}-i u \rho \varepsilon_{v}\right)^{2}+\varepsilon_{v}{ }^{2}\left(i u+u^{2}\right)}, \\
\psi_{ \pm}=\mp \kappa_{\lambda}+\xi \\
\xi=\sqrt{\kappa_{\lambda}^{2}-2 \varepsilon_{\lambda}^{2} \Lambda(u)}, \\
\quad \frac{p}{1-i u \eta_{u}}+\frac{q}{1+i u \eta_{d}} \\
-1-i u\left(\frac{p}{1-\eta_{u}}+\frac{q}{1+\eta_{d}}-1\right) .
\end{gathered}
$$


Proof. From (1), Feynman-Kac formula gives the following PIDE for the characteristic function,

$$
\begin{gathered}
-M_{\tau}+\left(r-\frac{1}{2} V-\lambda m\right) M_{x}+\frac{1}{2} V M_{x x}+\kappa_{v}\left(\theta_{v}-V\right) M_{v} \\
+\frac{1}{2} \varepsilon_{v}^{2} V M_{v v}+\rho \varepsilon V M_{x v}+\kappa_{\lambda}\left(\theta_{\lambda}-\lambda\right) M_{\lambda} \\
+\frac{1}{2} \varepsilon_{\lambda}^{2} \lambda M_{\lambda \lambda}+\kappa_{r}\left(\theta_{r}-r\right) M_{r}+\frac{1}{2} \varepsilon_{r}^{2} r M_{r r} \\
+\lambda \int_{-\infty}^{\infty}[M(X+y)-M] \omega(y) d y=0 \\
M(\Phi, X, r, V, \lambda, 0)=e^{\Phi X} .
\end{gathered}
$$

We conjecture that the solution of PDE (6) is

$$
\begin{aligned}
M & (\Phi, X, r, V, \lambda, \tau) \\
& =e^{X \Phi+r \tau \Phi+A(\Phi, \tau)+B(\Phi, \tau) V+C(\Phi, \tau)+D(\Phi, \tau) \lambda+E(\Phi, \tau)+F(\Phi, \tau) r},
\end{aligned}
$$

with initial conditions $A(\Phi, 0)=0, B(\Phi, 0)=0, C(\Phi, 0)=0$, $D(\Phi, 0)=0, E(\Phi, 0)=0$, and $F(\Phi, 0)=0$.

We consider the integral term in (6):

$$
\lambda \int_{-\infty}^{\infty}[M(X+y)-M] \omega(y) d y=\lambda M(\Phi, X, r, V, \lambda, \tau) U(\Phi),
$$

where $U(\Phi):=\int_{-\infty}^{\infty}\left(e^{\Phi y}-1\right) \omega(y) d y=p /\left(1-\Phi \eta_{u}\right)+q /(1+$ $\left.\Phi \eta_{d}\right)-1$.

Substituting (7) and (8) into (6) yields

$$
\begin{aligned}
& -A_{\tau}(\Phi, \tau)-B_{\tau}(\Phi, \tau) V-C_{\tau}(\Phi, \tau)-D_{\tau}(\Phi, \tau) \lambda \\
& -E_{\tau}(\Phi, \tau)-F_{\tau}(\Phi, \tau) r-\frac{1}{2} V \Phi-\lambda m \Phi \\
& +\frac{1}{2} V \Phi^{2}+\kappa_{v} \theta_{V} B(\Phi, \tau)-\kappa_{v} V B(\Phi, \tau) \\
& +\frac{1}{2} \varepsilon_{v}^{2} V B^{2}(\Phi, \tau)+\rho \varepsilon V \Phi B(\Phi, \tau) \\
& +\kappa_{r} \theta_{r} F(\Phi, \tau)-\kappa_{r} r F(\Phi, \tau)+\frac{1}{2} \varepsilon_{r}^{2} r F^{2}(\Phi, \tau) \\
& +\kappa_{\lambda} \theta_{\lambda} D(\Phi, \tau)-\kappa_{\lambda} \lambda D(\Phi, \tau)+\frac{1}{2} \varepsilon_{\lambda}^{2} \lambda D^{2}(\Phi, \tau) \\
& +\lambda U(\Phi)=0 .
\end{aligned}
$$

If we denote $\Lambda(\Phi):=U(\Phi)-m \Phi=p /\left(1-\Phi \eta_{u}\right)+q /(1+$ $\left.\Phi \eta_{d}\right)-1-\Phi\left(p /\left(1-\eta_{u}\right)+q /\left(1+\eta_{d}\right)-1\right)$, then (9) leads to a system of seven ordinary differential equations:

$$
\begin{gathered}
A_{\tau}(\Phi, \tau)+C_{\tau}(\Phi, \tau)+E_{\tau}(\Phi, \tau) \\
=\kappa_{v} \theta_{v} B(\Phi, \tau)+\kappa_{\lambda} \theta_{\lambda} D(\Phi, \tau)+\kappa_{r} \theta_{r} F(\Phi, \tau), \\
B_{\tau}(\Phi, \tau)=-\frac{1}{2}\left(\Phi-\Phi^{2}\right)-\left(\kappa_{v}-\rho \varepsilon \Phi\right) \\
\times B(\Phi, \tau)+\frac{1}{2} \varepsilon^{2} B^{2}(\Phi, \tau), \\
D_{\tau}(\Phi, \tau)=-\kappa_{\lambda} D(\Phi, \tau)+\frac{1}{2} \varepsilon_{\lambda}^{2} D^{2}(\Phi, \tau)+\Lambda(\Phi), \\
F_{\tau}(\Phi, \tau)=-k_{r} F(\Phi, \tau)+\frac{1}{2} \varepsilon_{r}^{2} F^{2}(\Phi, \tau), \\
A_{\tau}(\Phi, \tau)=k_{v} \theta_{v} B(\Phi, \tau), \\
C_{\tau}(\Phi, \tau)=k_{\lambda} \theta_{\lambda} D(\Phi, \tau), \\
E_{\tau}(\Phi, \tau)=k_{r} \theta_{r} F(\Phi, \tau) .
\end{gathered}
$$

We consider the second ODE (11), in which the general solution can be derived for the Riccati equation. Making the substitution,

$$
B(\Phi, \tau)=-\frac{2 O^{\prime}(\tau)}{\varepsilon^{2} O(\tau)}
$$

Hence,

$$
O^{\prime \prime}(\tau)+\left(k_{v}-\rho \varepsilon \Phi\right) O^{\prime}(\tau)-\frac{\varepsilon^{2}}{4}\left(\Phi-\Phi^{2}\right) O(\tau)=0 .
$$

The ODE (11) has a general solution of the form

$$
O(\tau)=C_{1} e^{(-1 / 2)_{\varphi_{-} \tau}}+C_{2} e^{(1 / 2)_{\varphi_{+} \tau}},
$$

where

$$
\begin{gathered}
\varphi_{ \pm}=\mp\left(k_{v}-\rho \varepsilon \Phi\right)+\varsigma, \\
\varsigma=\sqrt{\left(k_{v}-\rho \varepsilon \Phi\right)^{2}+\varepsilon^{2}\left(\Phi-\Phi^{2}\right)} .
\end{gathered}
$$

According to (15) and initial condition $C(\Phi, 0)=0, C_{1}$ and $C_{2}$ are two constants to be determined from boundary conditions,

$$
\begin{gathered}
O(0)=C_{1}+C_{2}, \\
O^{\prime}(0)=-\frac{1}{2} \varphi_{-} C_{1}+\frac{1}{2} \varphi_{+} C_{2} .
\end{gathered}
$$

We obtain that the solutions of (21) is

$$
\begin{aligned}
& C_{1}=\frac{\varphi_{+} O(0)}{2 \varsigma}, \\
& C_{2}=\frac{\varphi_{-} O(0)}{2 \varsigma} .
\end{aligned}
$$


Therefore, the exact solution of (11) is

$$
B(\Phi, \tau)=-\left(\Phi-\Phi^{2}\right) \frac{1-e^{-\varsigma \tau}}{\varphi_{-}+\varphi_{+} e^{-\zeta \tau}} .
$$

Substituting (23) into (14) and using initial conditions $A(\Phi, 0)=0$, we have

$$
A(\Phi, \tau)=-\frac{\kappa_{v} \theta_{v}}{\varepsilon^{2}}\left(\varphi_{+} \tau+2 \ln \left[\frac{\varphi_{-}+\varphi_{+} e^{-\varsigma \tau}}{2 \varsigma}\right]\right)
$$

The OEDs for $C(\Phi, \tau), D(\Phi, \tau), E(\Phi, \tau)$, and $F(\Phi, \tau)$ are solved by analogy. Hence

$$
\begin{gathered}
C(\Phi, \tau)=-\frac{\kappa_{\lambda} \theta_{\lambda}}{\varepsilon^{2}}\left(\psi_{+} \tau+2 \ln \left[\frac{\psi_{-}+\psi_{+} e^{-\xi \tau}}{2 \xi}\right]\right), \\
D(\Phi, \tau)=2 \Lambda(\Phi) \frac{1-e^{-\xi \tau}}{\psi_{-}+\psi_{+} e^{-\xi \tau}}, \\
E(\Phi, \tau)=0 \\
F(\Phi, \tau)=0 \\
\psi_{ \pm}=\mp \kappa_{\lambda}+\xi \\
\xi=\sqrt{\kappa_{\lambda}^{2}-2 \varepsilon_{\lambda}^{2} \Lambda(\Phi)}
\end{gathered}
$$

Using $\phi(u)=M(i u)$, it is easy to get Theorem 1 .

\section{Power Option Pricing Using the Fast Fourier Transform}

4.1. Power Option. Under the risk-neutral measure $\mathbb{Q}$, the price of the power call option $C(S, r, V, \lambda, t)$ at time $t$ with strike price $K$ and maturity date $T$ is given by

$$
C(S, r, V, \lambda, t)=E^{\mathbb{Q}}\left[e^{-\int_{t}^{T} r_{u} d u}\left(S^{\beta}-K\right)^{+}\right] .
$$

As a European option, the valuation of power at initial time is

$$
c(K, T, \beta):=C(S, r, V, \lambda, 0)=E^{\mathbb{Q}}\left[e^{-\int_{0}^{T} r_{u} d u}\left(S^{\beta}-K\right)^{+}\right] .
$$

Remark 2. In particularly, when $\beta=1$, the power option degenerates into the vanilla option.

4.2. Fast Fourier Transform. Let $k$ denote the log of the strike price $K$, and let $c(k)$ be the desired value of an initial power call option with strike price $e^{k}$. The risk-neutral density of the price $X(T)$ at time $T$ is $q_{T}(X)$. From (27), the initial value of power call option is related to the risk-neutral density $q_{T}(X)$ by

$$
c(k)=e^{-\int_{0}^{T} r_{u} d u} \int_{k}^{\infty}\left(e^{\beta X(T)}-e^{k}\right) q_{T}(X) d X .
$$

Here we employ the price of a zero coupon bond $Q(T)$ at time $T$ to express the stochastic discounted process $e^{-\int_{0}^{T} r_{u} d u}$

$$
Q(T)=P(T) e^{-H(T) r_{0}},
$$

where

$$
\begin{gathered}
P(T)=\left[\frac{2 m e^{(T / 2)\left(\kappa_{r}+m\right)}}{\left(\kappa_{r}+m\right)\left(e^{m T}-1\right)+2 m}\right]^{2 \kappa_{r} \theta_{r} / \varepsilon_{r}^{2}}, \\
H(T)=\frac{2\left(e^{m T}-1\right)}{\left(\kappa_{r}+m\right)\left(e^{m T}-1\right)+2 m}, \\
m=\sqrt{\kappa_{r}^{2}+2 \varepsilon_{r}^{2}} .
\end{gathered}
$$

Then (28) can be written as

$$
c(k)=Q(T) \int_{k}^{\infty}\left(e^{\beta X(T)}-e^{k}\right) q_{T}(X) d X .
$$

Note that $c(k)$ tends to $S_{0}^{\beta}$ as $k$ tends to $-\infty$, and hence the call pricing function (31) is not square integrable. According to Wong and Lo [15], we now consider the modified call price function $\bar{c}(k)$ defined by

$$
\bar{c}(k) \equiv e^{\alpha k} c(k), \quad \text { for } \alpha>0 .
$$

The Fourier transform of $\bar{c}(k)$ is defined by

$$
\begin{aligned}
\omega_{T} & (u) \\
& =\int_{-\infty}^{\infty} e^{i u k} \bar{c}(k) d k \\
& =\int_{-\infty}^{\infty} e^{i u k} e^{\alpha k} c(k) d k \\
& =\int_{-\infty}^{\infty} e^{i u k} e^{\alpha k} Q(T) \int_{k}^{\infty}\left(e^{\beta X}-e^{k}\right) q_{T}(X) d X d k \\
& =Q(T) \int_{-\infty}^{\infty} q_{T}(X) \int_{-\infty}^{X}\left(e^{\beta X+\alpha k}-e^{(1+\alpha) k}\right) e^{i u k} d k d X \\
& =Q(T) \int_{-\infty}^{\infty} e^{-r T} q_{T}(X)\left(\frac{e^{(\beta+\alpha+i u) X}}{\alpha+i u}-\frac{e^{(1+\alpha+i u) X}}{1+\alpha+i u}\right) d X \\
& =Q(T)\left[\frac{\phi_{T}(u-(\beta+\alpha) i)}{\alpha+i u}-\frac{\phi_{T}(u-(1+\alpha) i)}{1+\alpha+i u}\right] .
\end{aligned}
$$

Then the inverse transform of $\omega_{T}(u)$ is given by

$$
\bar{c}(k)=\frac{1}{\pi} \int_{0}^{\infty} e^{-i u k}{\omega_{T}}(u) d u .
$$

Then

$$
c(k)=\frac{e^{-\alpha k}}{\pi} \int_{0}^{\infty} e^{-i u k} \omega_{T}(u) d u .
$$


Using the Trapezoid rule for the integral in (35), the value of $c(k)$ is approximated as

$$
c(k) \approx \frac{e^{-\alpha k}}{\pi} \sum_{j=0}^{N} e^{-i u k} \varpi_{T}\left(u_{j}\right) \nu,
$$

where $u_{j}=\nu(j-1)$

The FFT returns $N$ values of $k$ and for a regular spacing size of $z$ where $N$ is a power of 2 . The value for $k$ is

$$
k_{u}=-b+z(a-1), \quad \text { for } a=1, \ldots, N,
$$

which corresponds to log strike prices ranging from $-b$ to $b$, where $b=N z / 2$.

Substituting (37) into (36) yields

$$
c\left(k_{u}\right) \approx \frac{e^{-\alpha k_{u}}}{\pi} \sum_{j=0}^{N} e^{-i z v(j-1)(a-1)} e^{i b u_{j}} \varpi_{T}\left(u_{j}\right) v
$$

here we denote $v z=2 \pi / N$.

With Simpson's rule weightings, the value of European call option is

$$
\begin{gathered}
c\left(k_{u}\right) \approx \\
\frac{e^{-\alpha k_{u}}}{\pi} \sum_{j=1}^{N} e^{-i(2 \pi / N)(j-1)(a-1)} e^{i b u_{j}}{\Phi_{T}} \\
\times\left(u_{j}\right) \frac{\nu}{3}\left[3+(-1)^{j}-\ell_{j-1}\right],
\end{gathered}
$$

where

$$
\ell_{n}= \begin{cases}1, & n=0, \\ 0, & \text { otherwise }\end{cases}
$$

\section{Numerical Examples and Discussion}

In this section, we present several numerical experiments to compare numerical differences between the FFT and Monte Carlo simulation and discuss the effects of power coefficient. Now, we perform some Monte Carlo simulations for the endowment dynamics by using the discrete scheme of system (1) as follows:

$$
\begin{aligned}
\ln S(t+\Delta t)= & \ln S(t)+\left(r-d-\lambda(t) m-\frac{1}{2} V(t)\right) \Delta t \\
& +\sqrt{V(t)} d W^{s}(t) \\
& +\left(e^{y}-1\right) \ln S(t)(N(t+\Delta t)-N(t)), \\
V(t+\Delta t)=V(t) & +\kappa_{v}\left(\theta_{v}-V(t)\right) \Delta t+\varepsilon_{v} \sqrt{V(t)} d W^{v}(t), \\
\lambda(t+\Delta t)=\lambda(t) & +\kappa_{\lambda}\left(\theta_{\lambda}-\lambda(t)\right) d t+\varepsilon_{\lambda} \sqrt{\lambda(t)} d W^{\lambda}(t), \\
r(t+\Delta t)= & r(t)+\kappa_{r}\left(\theta_{r}-V(t)\right) \Delta t+\varepsilon_{r} \sqrt{r(t)} d W^{r}(t),
\end{aligned}
$$

where $d W^{s}(t)=\varepsilon_{1} \sqrt{\Delta t}, d W^{v}(t)=\rho \varepsilon_{1} \sqrt{\Delta t}, d W^{\lambda}(t)=$ $\varepsilon_{2} \sqrt{\Delta t}$, and $d W^{r}(t)=\varepsilon_{3} \sqrt{\Delta t} ; \Delta t$ is the time interval; $\varepsilon_{1}, \varepsilon_{2}$,
TABle 1: Power option prices in $\beta=0.98$ case: FFT versus Monte Carlo.

\begin{tabular}{lccc}
\hline Strike price & FFT & Monte Carlo & \% difference \\
\hline 90.2830 & 6.0740 & 6.0725 & 0.0246 \\
92.1938 & 5.4487 & 5.448 & 0.0136 \\
94.1451 & 4.8635 & 4.8624 & 0.0218 \\
96.1377 & 4.3188 & 4.3212 & -0.0544 \\
98.1724 & 3.8143 & 3.8137 & 0.0161 \\
100.2502 & 3.3498 & 3.3505 & -0.0223 \\
102.3720 & 2.9244 & 2.9242 & 0.0066 \\
104.5387 & 2.5371 & 2.538 & -0.035 \\
106.7512 & 2.1867 & 2.1859 & 0.035 \\
107.8750 & 2.0249 & 2.0235 & 0.0693 \\
110.1581 & 1.7269 & 1.7255 & 0.0824 \\
\hline
\end{tabular}

TABLE 2: Power option prices in $\beta=1$ case: FFT versus Monte Carlo.

\begin{tabular}{lccc}
\hline Strike price & FFT & Monte Carlo & \% difference \\
\hline 90.2830 & 10.7797 & 10.7799 & -0.002 \\
92.1938 & 9.9356 & 9.9344 & 0.0117 \\
94.1451 & 9.1277 & 9.1266 & 0.0126 \\
96.1377 & 8.3574 & 8.3575 & -0.0013 \\
98.1724 & 7.6256 & 7.6263 & -0.0095 \\
100.2502 & 6.9334 & 6.936 & -0.0373 \\
102.3720 & 6.2811 & 6.2804 & 0.0106 \\
104.5387 & 5.6691 & 5.6693 & -0.0033 \\
106.7512 & 5.0974 & 5.0973 & 0.0016 \\
107.8750 & 4.8265 & 4.8246 & 0.0401 \\
110.1581 & 4.3145 & 4.3141 & 0.0102 \\
\hline
\end{tabular}

and $\varepsilon_{3}$ are samples from the standard normal distribution with correlation coefficient $0 ; N(t)=j$, if $S_{j} \leq t \leq S_{j+1}$, $S_{j}=\sum_{k=1}^{j} \tau_{k}, \tau_{k}$ is the $k$ th jump interval which obeys an exponential distribution with parameter $\lambda$.

The option prices are calculated by Monte Carlo simulation using

$$
\begin{aligned}
& V(S(t), K, V(t), \lambda(t), r(t), t) \\
& \quad=E^{\mathbb{Q}}\left[\left(\frac{S_{T}^{\varepsilon}+S_{T}^{\varepsilon-}}{2}-K\right)^{+} \mid F_{t}\right] .
\end{aligned}
$$

In our numerical examples by the FFT, we take $N=4098$ and $v=600 / N$ which lead to the log strike spacing of $z=\pi / 300$ and the damping coefficient $\alpha=1.18$. Other parameters are denoted as $r_{0}=0.05, \kappa_{v}=0.3, \theta_{v}=0.15$, $\varepsilon_{v}=0.1, \kappa_{\lambda}=5, \theta_{\lambda}=0.6, \varepsilon_{\lambda}=0.3, \kappa_{r}=0.5, \theta_{r}=0.05$, $\varepsilon_{r}=0.1, \rho=-0.25, \eta_{u}=0.03, \eta_{d}=0.13, p=0.4, q=0.6$, $S_{0}=100, V_{0}=0.15, \lambda_{0}=3, T=0.5, \kappa=100$, and $\Delta t=0.01$. The results are presented in Tables 1,2 , and 3 .

Numerical examples presented that the FFT is faster than Monte Carlo method with different parameters. To different strike prices, the FFT takes around approximate 340 seconds to produce 4098 option prices in three numerical experiments. However, Monte Carlo technique takes around 1260 seconds. On the level of the accuracy, comparing with 
TABLE 3: Power option prices in $\beta=1.02$ case: FFT versus Monte Carlo.

\begin{tabular}{lccc}
\hline Strike price & FFT & Monte Carlo & \% difference \\
\hline 90.2830 & 15.9551 & 15.9556 & -0.0031 \\
92.1938 & 14.8717 & 14.8715 & 0.0012 \\
94.1451 & 13.81993 & 13.8197 & 0.0014 \\
96.1377 & 12.8024 & 12.8038 & -0.0111 \\
98.1724 & 11.8216 & 11.8219 & -0.0025 \\
100.2502 & 10.8797 & 10.8799 & -0.0018 \\
102.3720 & 9.9786 & 9.9802 & -0.0159 \\
104.5387 & 9.1201 & 9.1193 & 0.0088 \\
106.7512 & 8.3053 & 8.306 & -0.0084 \\
107.8750 & 7.9147 & 7.9155 & -0.0105 \\
110.1581 & 7.1675 & 7.1673 & 0.0034 \\
\hline
\end{tabular}

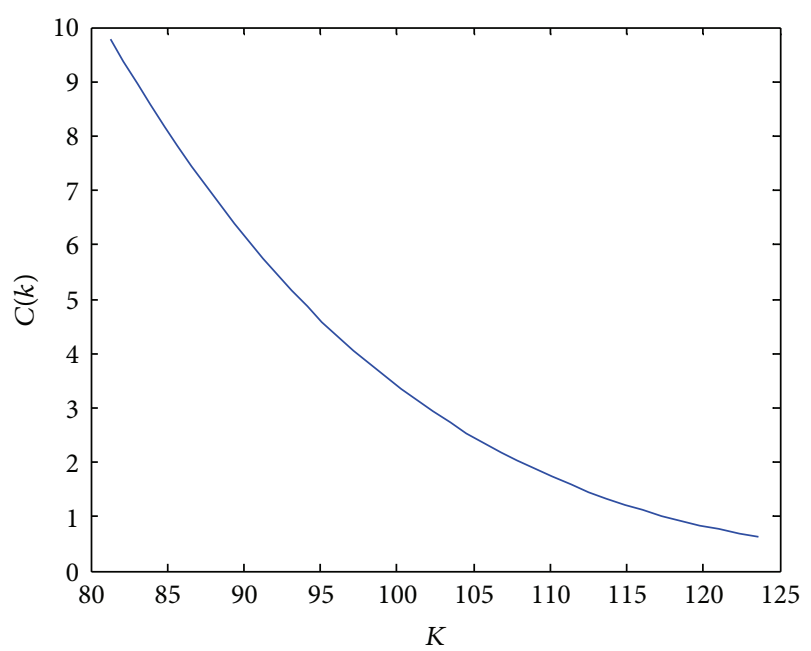

Figure 1: Power option prices in $\beta=0.98$ case.

Monte Carlo, we show that the percentage differences of the FFT are less than $0.1 \%$ in Tables 1,2 , and 3 . Therefore, the FFT is more accurate and faster than Monte Carlo.

To examine effects of the power coefficient $\beta$, we calculate valuations with $\beta=0.98, \beta=1$, and $\beta=1.02$. Firstly, we can see that with the $\beta$ rising, the gaps between $S^{\beta}$ and $K$ become greater in Figures 1, 2, and 3. Secondly, accordoing to different $\beta$, we find that valuations of option are increasing with the growth of $\beta$. It is because the fluctuations and risks of the underlying asset price are enhancing with the growth of $\beta$, and in order to compensate risk, the option seller need gets higher price of option. It means that the seller can design different power options with different risks or prices by defining different the power coefficients. Therefore, power option is one of convenient options which are designed easily.

\section{Conclusion}

In this paper, we study a more general and realistic double exponential-jump model which links the stochastic interest rate, volatility, and jump intensity. If we decide the constant

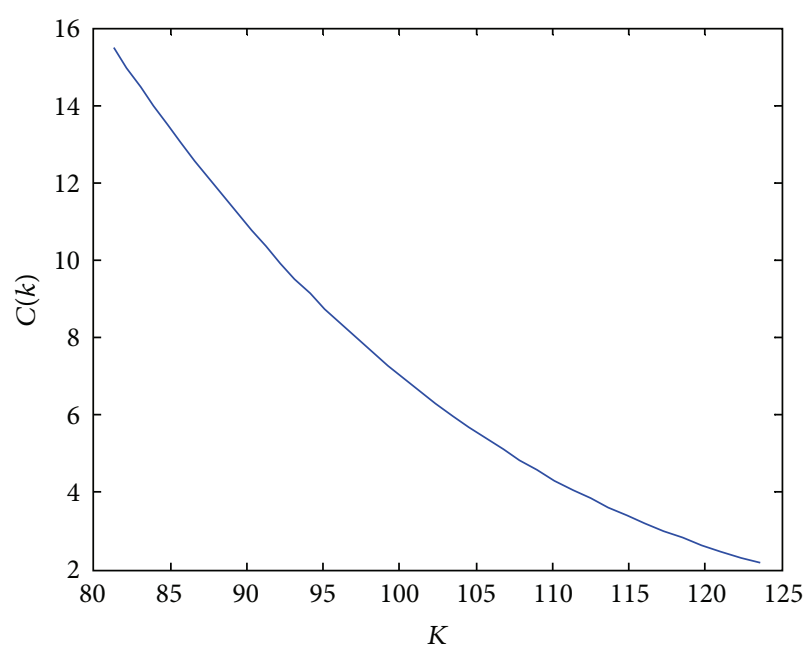

Figure 2: Power option prices in $\beta=1$ case.

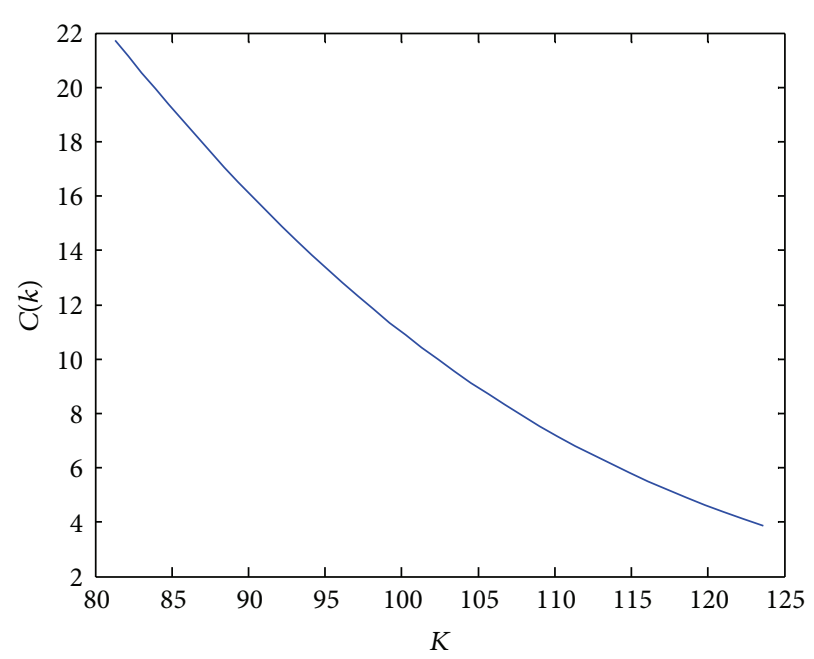

Figure 3: Power option prices in $\beta=1.02$ case.

interest rate, volatility, or jump intensity, our model can be degenerated into many previous models, for example, Black and Scholes' model in [1], Santa-Clara and Yan' model in [4, 5], Carr and Madan's model in [14], Zhang and Wang's model in [10, 11], and Zhang and Wang's model in [17].

Moreover, we obtain the characteristic function of the log asset price, which is important to not only take the FFT in [14-17] but also derive the option pricing formula, for example, in [18]. Then, in our paper, a power option is defined and its valuations are computed with the FFT by characteristic function. Through numerical examples, comparing the results produced from the FFT and the Monte Carlo simulations of power option valuation, we find that the FFT is approximate with Monte Carlo approach but has better stability, higher accuracy, and faster speed in pricing power option. In addition, we show that the seller can design different power options with different risks or prices by defining different the power coefficients. 


\section{Acknowledgment}

This work is supported by the Fundamental Research Funds for the Central Universities (JBK130401).

\section{References}

[1] F. Black and M. Scholes, "The pricing of options and corporate liabilities," The Journal of Political Economy, vol. 81, no. 3, pp. 637-654, 1973.

[2] K. Chen and S. H. Poon, "Variance swap premium under stochastic volatility and self-exciting jumps," SSRN, 2200172, 2013.

[3] R. Cont and P. Tankov, Financial Modelling with Jump Processes, Financial Mathematics, Chapman \& Hall, Boca Raton, Fla, USA, 2004.

[4] P. Santa-Clara and S. Yan, "Crashes, volatility, and the equity premium: Lessons from S\&P 500 options," Review of Economics and Statistics, vol. 92, no. 2, pp. 435-451, 2010.

[5] P. Santa-Clara and S. Yan, "Jump and volatility risk and risk premia: a new model and lessons from S\&P 500 options," National Bureau of Economic Research, no. w10912, 2004.

[6] A. Amin, "Hybrid equity, FX, and interest rate models with stochastic volatility and jump diffusion," FX, and Interest Rate Models with Stochastic Volatility and Jump Diffusion, 2010.

[7] C. Chang, C. D. Fuh, and S. K. Lin, "A tale of two regimes: theory and empirical evidence for a Markov-modulated jump diffusion model of equity returns and derivative pricing implications," Journal of Banking \& Finance, vol. 37, no. 8, pp. 3204-3217, 2013.

[8] J. P. F. Charpin and M. Cummins, "Fast Fourier transform option pricing: efficient approximation methods under multifactor stochastic volatility and jumps," Topics in Numerical Methods for Finance, vol. 19, pp. 115-137, 2012.

[9] P. G. Zhang, "An introduction to exotic options," European Financial Management, vol. 1, no. 1, pp. 87-95, 1995.

[10] K. Zhang and S. Wang, "Pricing options under jump diffusion processes with fitted finite volume method," Applied Mathematics and Computation, vol. 201, no. 1-2, pp. 398-413, 2008.

[11] K. Zhang and S. Wang, "A computational scheme for options under jump diffusion processes," International Journal of Numerical Analysis and Modeling, vol. 6, no. 1, pp. 110-123, 2009.

[12] P. J. Green, "Reversible jump Markov chain Monte Carlo computation and Bayesian model determination," Biometrika, vol. 82, no. 4, pp. 711-732, 1995.

[13] P. Glasserman, Monte Carlo Methods in Financial Engineering, vol. 53, Springer, New York, NY, USA, 2004.

[14] P. Carr and D. Madan, "Option valuation using the fast Fourier transform," Journal of Computational Finance, vol. 2, no. 4, pp. 61-73, 1999.

[15] H. Y. Wong and Y. W. Lo, "Option pricing with mean reversion and stochastic volatility," European Journal of Operational Research, vol. 197, no. 1, pp. 179-187, 2009.

[16] E. Pillay and J. G. O'Hara, "FFT based option pricing under a mean reverting process with stochastic volatility and jumps," Journal of Computational and Applied Mathematics, vol. 235, no. 12, pp. 3378-3384, 2011.

[17] S. Zhang and L. Wang, "Fast Fourier transform option pricing with stochastic interest rate, stochastic volatility and double jumps," Applied Mathematics and Computation, vol. 219, no. 23, pp. 10928-10933, 2013.

[18] A. L. Lewis, Option Valuation under Stochastic Volatility, Finance Press, Newport Beach, Calif, USA, 2000. 


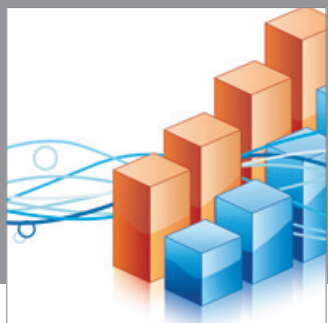

Advances in

Operations Research

mansans

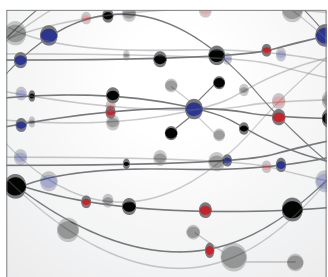

The Scientific World Journal
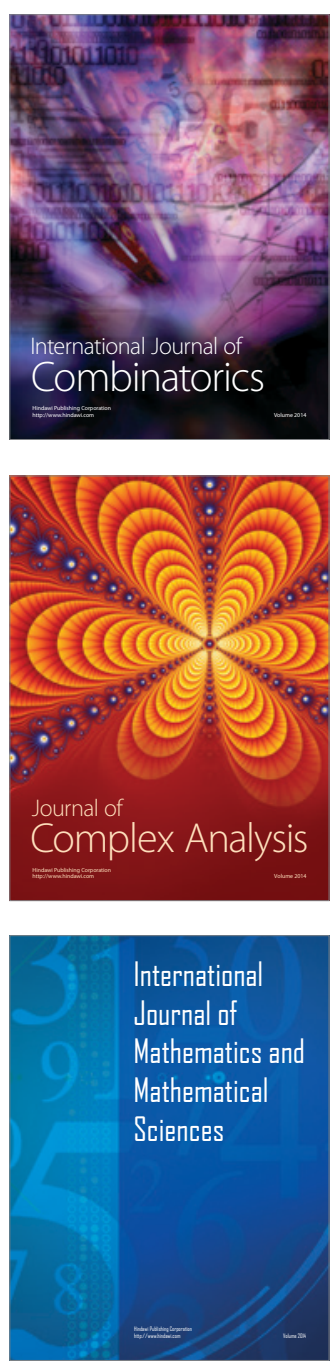
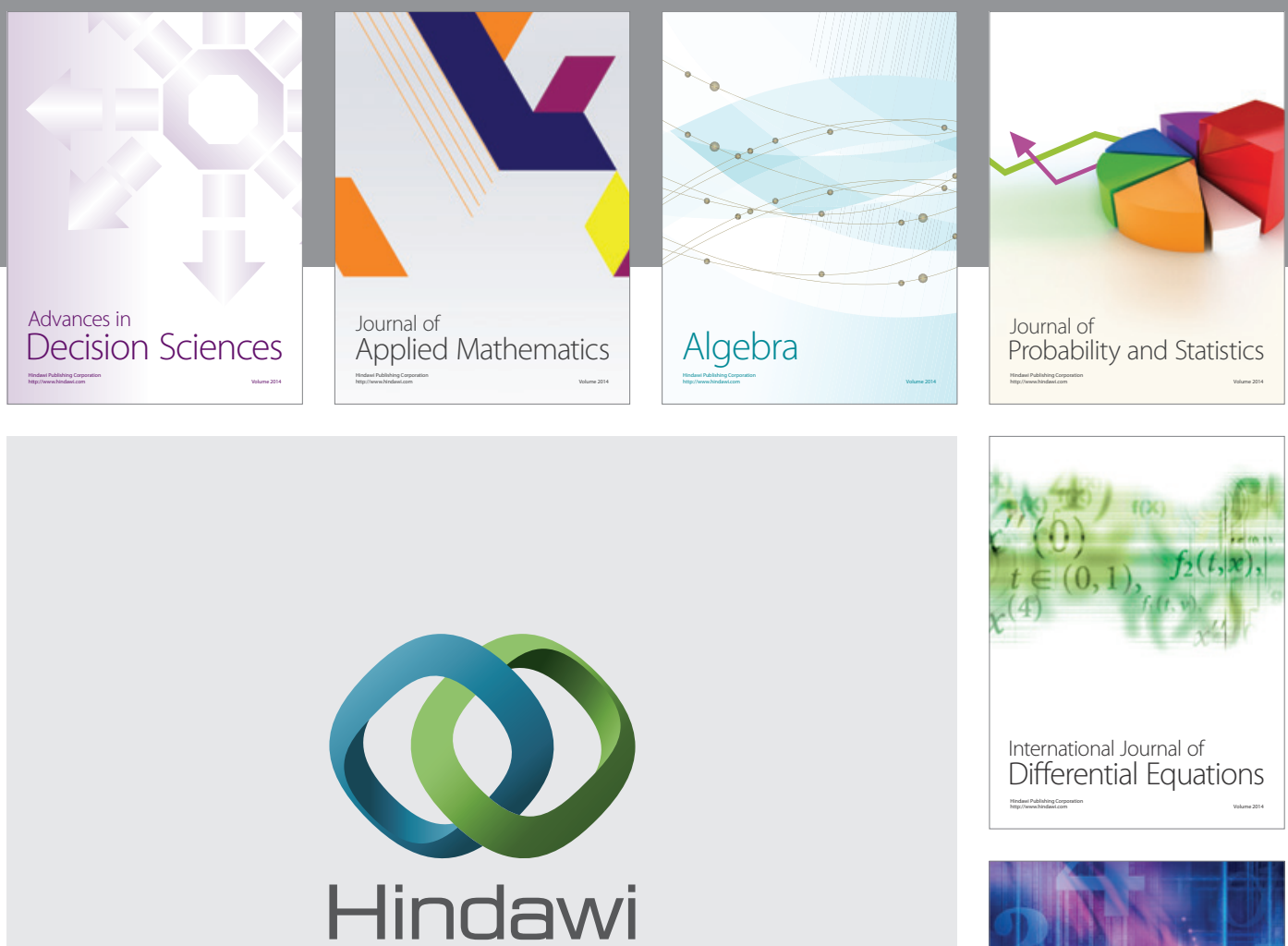

Submit your manuscripts at http://www.hindawi.com
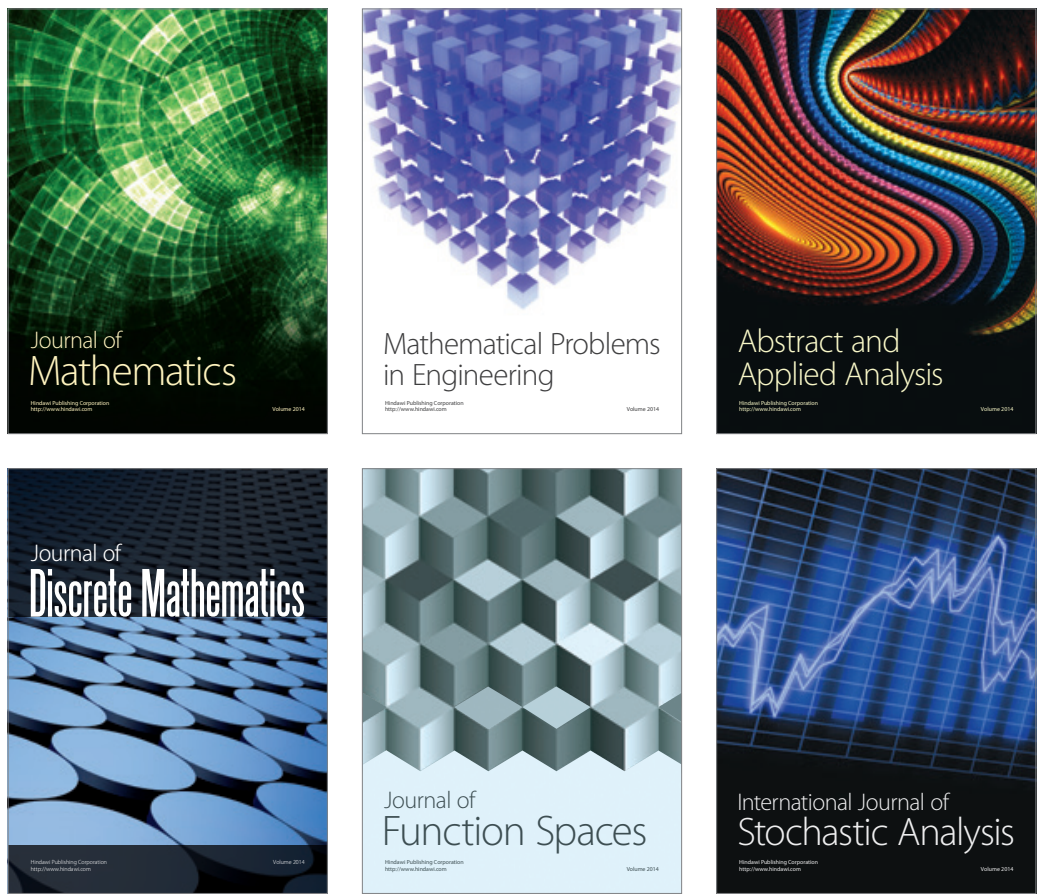

Journal of

Function Spaces

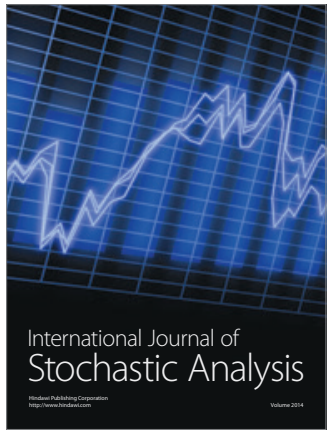

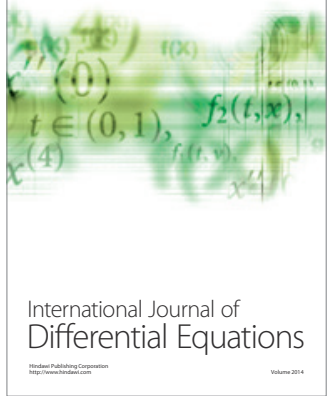
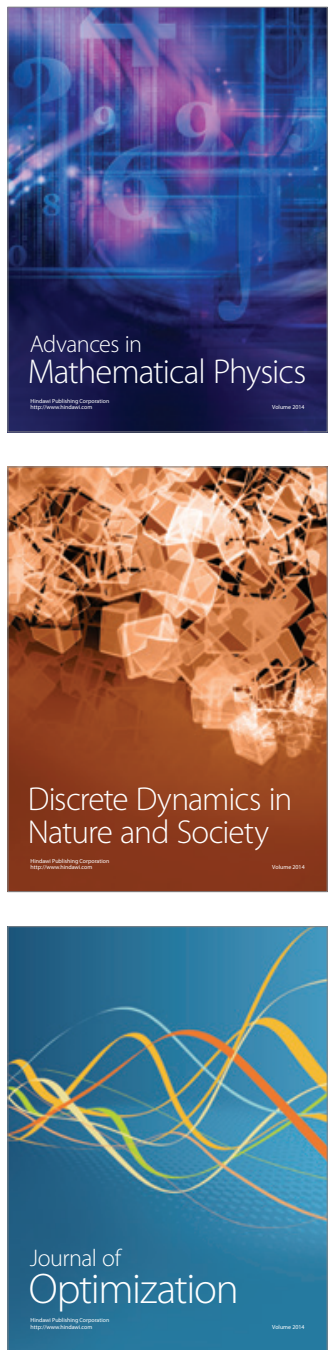